\title{
Analysis of Corrugated Bulkhead against Transverse Plane Bulkhead in a Tanker Ship
}

\author{
Hamzah, ${ }^{\mathrm{a}, *}$ Ganding Sitepu, ${ }^{\mathrm{b}}$ Muh. Hidayat Arif, ${ }^{\mathrm{c}}$ \\ ${ }^{a}$ Department of Naval Engineering, Faculty of Engineering, Hasanuddin University. Email: anca_nava199@yahoo.com \\ bDepartment of Naval Engineering, Faculty of Engineering, Hasanuddin University. Email: sitepu.unhas@gmail.com \\ 'Department of Naval Engineering, Faculty of Engineering, Hasanuddin University.
}

\begin{abstract}
Ship is built from thousands of integrated construction materials. The selection of a strong and efficient construction material is significant since it directly affects the weight of the ship. For a watertight bulkhead in a tanker ship, there are two kinds of bulkhead construction which are transverse plane bulkhead and corrugated bulkhead. The selection of these two bulkhead constructions for watertight bulkhead is based on the bulkhead strength analysis as well as bulkhead weight. The analysis was conducted using an engineering structural analysis software by comparing the bulkhead structural configurations which has the same von Misses tension. For corrugated bulkhead, corrugation angle was simulated between $45^{\circ}-90^{\circ}$ while for transverse plane bulkhead, the range of stiffener distances was simulated between $400-1400 \mathrm{~mm}$. The result shows that minimum corrugated bulkhead weight which is 41.18 ton can be obtained on the corrugation angle of $60^{\circ}$ with the structural efficiency ratio of $1.79 \%-30.91 \%$ while for transverse plane bulkhead, minimum weight of bulkhead which is 71.175 ton can be obtained on the stiffeners distance of $600 \mathrm{~mm}$ with structural efficiency ratio of $2.62 \%-60.25 \%$. It can be seen that for the same von Misses tension, corrugated bulkhead construction is more efficient in terms of bulkhead weight and cost with the difference weights of almost 30 ton.
\end{abstract}

Keywords: Corrugated bulkhead; tanker ship; transverse plane bulkhead; von Misses tension

\section{Introduction}

The construction configuration and material must be carefully selected when designing ship construction. The common selection criteria are the construction strength to fulfil the ship strength requirements as well as the construction weight to reduce the ship building cost. One example of the selection of construction configuration is the selection of watertight bulkhead construction configuration for a tanker ship. Watertight bulkhead is a bulkhead constructed in a ship to divide ship into compartments. It has to be watertight to prevent water to flow to the nearest compartments. In a tanker cargo tank, the watertight bulkhead is constructed to prevent one liquid cargo to mix up with other different liquid cargo especially with different density and viscosity. The watertight bulkhead is constructed transversally and longitudinally in the tanker cargo tank. There are two kinds of watertight bulkheads

\footnotetext{
${ }^{*}$ Corresponding author. Tel.: +62-812-4420-9266

Jalan Poros Malino km. 6, Bontomarannu

Gowa, Sulawesi Selatan, Indonesia, 92171
}

which are being used in a tanker ship namely transverse plane bulkhead and corrugated bulkhead.

The selection of the design watertight bulkhead construction is based on the cargo hold capacity maximization as well as for easy and reduced cargo tank cleaning process time. In addition to the parameters, the bulkhead selection is also considered the ease of tank operational process, construction structural strength and the economic aspect of the selected bulkhead.

In the ship construction rules, the use of transverse plane bulkhead or corrugated bulkhead as transversal bulkhead is not regulated. Furthermore, for corrugated bulkhead, the plate geometry is not fully regulated in the construction rules. The rules only regulate the corrugation depth, angle and the radius between corrugations. Even, for some classification bureaus, the rules for corrugation angle are different. Therefore, there is no exact rule for determining a good geometry form of corrugated bulkhead.

In the construction application, both types of the bulkhead are being used interchangeably or being used together on the same ship. As in a tanker ship which is being the case study for this research, the watertight bulkhead to 
separate between cargo hold are constructed using both types of the bulkheads. Transversal bulkhead uses corrugated bulkhead with the plate thickness of $14 \mathrm{~mm}$ while longitudinal bulkhead uses transverse plane bulkhead with the same thickness. An analysis for comparing the two forms of the watertight bulkhead in a tanker ship is conducted in order to identify the most efficient use of those two bulkheads in terms of some criteria as explained previously.

The tanker ship which is under investigation is owned by one of the oil company in Indonesia which is still under construction in a shipyard. Material used for cargo hold tank is Steel Grade A while the ship itself is 17,500 LTDWT.

\section{Theoretical background}

\subsection{Transverse plane bulkhead}

This type of watertight bulkhead construction is the most common bulkhead construction being used in a ship. This bulkhead is constructed by installing a plane plate with a required plate thickness in a longitudinal or transversal position in a ship. This plate then be strengthened with stiffeners using angle bar, $\mathrm{T}$ bar and bulb plate (Fig. 1). Based on Indonesian Bureau Classification (BKI) Vol. II, Rules for Hull, Section 9/A.1.1 [1], the distance between stiffeners is no less than the distance between ship frames or no less than $600 \mathrm{~mm}$.

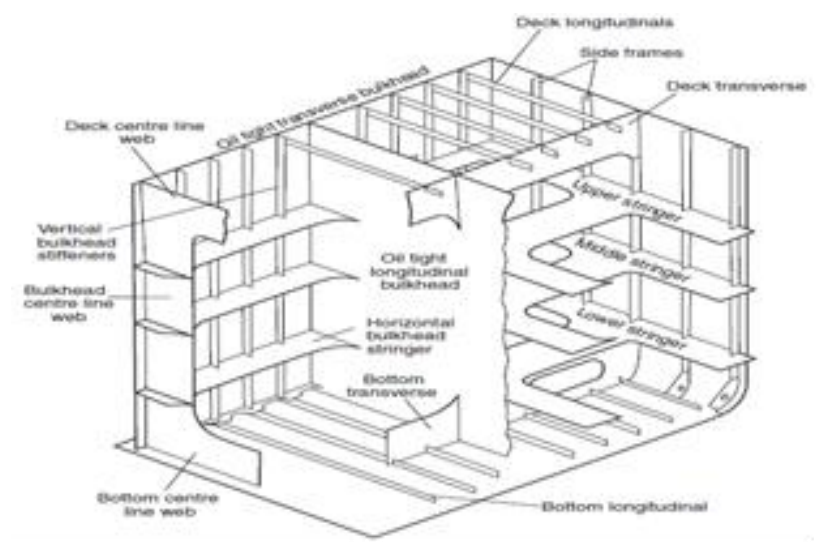

Figure 1. Transversal watertight bulkhead using transverse plane bulkhead [2]

\subsection{Corrugated Bulkhead}

Different cargo will require different packaging and cargo hold shape. For liquid cargo such as oil in a tanker ship, the separation of cargo hold is compulsory. Corrugated watertight bulkhead is commonly used to separate between tanks. The main reason for selecting this bulkhead is for maintenance reason as it is relatively easy to clean up the tank. This type of bulkhead does not use stiffeners as the corrugation form replaces the stiffener tasks. The corrugation in this bulkhead is made vertically (Fig. 2) in order to increase the bulkhead effectivity in maintaining liquid density as well as supporting structural strength [3].

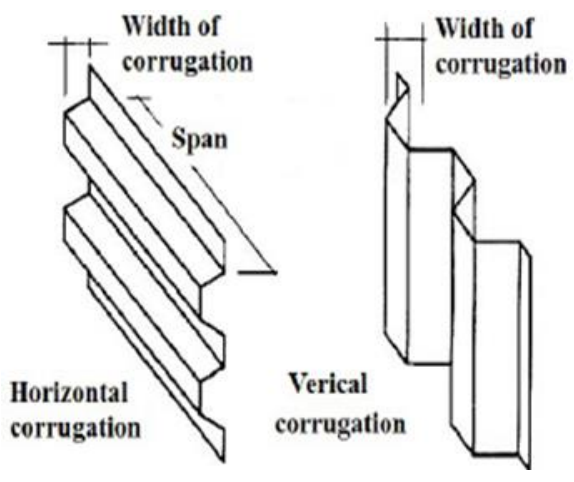

Figure 2. Horizontal and vertical corrugated bulkhead [4]

In a corrugation plate, the main parameter for determining bulkhead structural strength is the value of bulkhead corrugation angle. The construction of corrugated bulkhead can be seen in Fig. 3 .

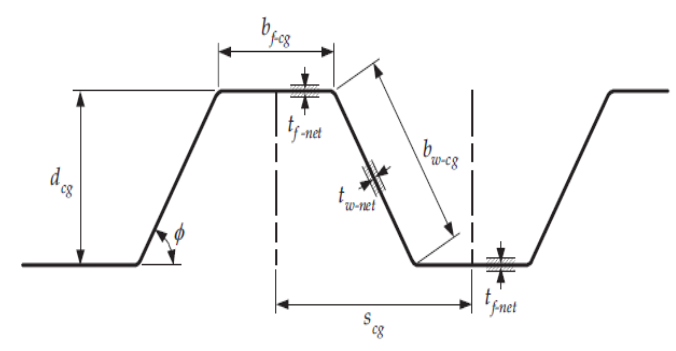

Figure 3. Element of the corrugated bulkhead [5]

where:

$s_{c g} \quad=$ length of the half wave, in $\mathrm{mm}$

$b_{w-c g}=$ width of the web plate, in $\mathrm{mm}$

$b_{f-c g}=$ width of the face plate, in $\mathrm{mm}$

$d_{c g}=$ depth of the corrugated bulkhead, in $\mathrm{mm}$

$=\mathrm{b}_{\mathrm{w}-\mathrm{cg}} \sin \theta$

$t_{f-n e t}=$ thickness of the face plate, in $\mathrm{mm}$

$t_{w-n e t}=$ thickness of the web plate, in $\mathrm{mm}$

$\theta \quad=$ angle of the bulkhead corrugation $\left(\geq 55^{\circ}\right)$

\subsection{Optimization theory}

There are three parameters in optimization theory which are [6]:

1. Design variable, the values are changing during optimization process.

2. Constraints, the values are constant during the optimization process.

3. Objectives, contains criteria for the optimization process.

\section{Research methodology}

The analysis consists of three main steps. The first step is determining variables and their values for optimization. The variables will be based on the parameters which have been explained in the previous section. The second step is determining sloshing pressure in the tank. The main purpose of this step is to determine pressure as a result of longitudinal 
liquid motion in the tank. The last step is determining additional load of construction and equipment weight.

\subsection{Variables for optimization}

The main variable for the optimization process in corrugated bulkhead is the corrugation angle. As the asymmetry principle, the other bulkhead dimension will be changed as well. The optimization variables for corrugated bulkhead in this research can be seen in Table 1 .

Table 1. Variable for optimization of corrugated bulkhead structure

\begin{tabular}{|c|c|c|c|}
\hline No & $\begin{array}{l}\text { Optimization } \\
\text { variables }\end{array}$ & & Description \\
\hline \multirow{5}{*}{1} & \multirow{5}{*}{ Design variables } & 1 & Angle of the corrugation bulkhead $(\theta)$ \\
\hline & & 2 & Length of the half wave $\left(s_{c g}\right)$ \\
\hline & & 3 & Width of the web plate $\left(b_{w-c g}\right)$ \\
\hline & & 4 & Width of the plate $\left(b_{f c c}\right)$ \\
\hline & & 5 & Thickness o the corrugated bulkhead $(t)$ \\
\hline \multirow{3}{*}{2} & \multirow{3}{*}{ Constraints } & 1 & Depth of the corrugated bulkhead $\left(d_{c g}\right)$ \\
\hline & & 2 & Working load on the corrugated \\
\hline & & 3 & Working stress \\
\hline 3 & Objectives & 1 & Minimum bulkhead weight \\
\hline
\end{tabular}

In transverse plane bulkhead, the main variable for the optimization process is the distance between stiffeners. The other variables for this bulkhead can be seen in Table 2 .

For corrugated bulkhead, the existing angle of corrugation is $64^{\circ}$. Based on this initial value, the corrugation angle then is varied in order to find the optimum angle. Hence, the design variables values for the corrugated bulkhead can be seen in Table 3 .

While for transverse plane bulkhead, the design variable values can be seen in Table 4 .

Table 2. Variable for optimization of transverse plane bulkhead

\begin{tabular}{|c|c|c|c|}
\hline No & $\begin{array}{c}\text { Optimization } \\
\text { variables }\end{array}$ & & Description \\
\hline 1 & Design variables & $\begin{array}{l}2 \\
3 \\
\end{array}$ & $\begin{array}{l}\text { Transverse plane bulkhead plate } \\
\text { thickness } \\
\text { The distance between stiffeners } \\
\text { Bulkhead construction } \\
\end{array}$ \\
\hline 2 & Constraints & 1 & $\begin{array}{l}\text { Depth of the corrugated bulkhead }\left(d_{c g}\right) \\
\text { Working load on the corrugated } \\
\text { bulkhead } \\
\text { Working stress }\end{array}$ \\
\hline 3 & Objectives & 1 & Minimum bulkhead weight \\
\hline
\end{tabular}

Table 3. Design variable values for corrugated bulkhead with varied corrugated angles

\begin{tabular}{cccc}
\hline$\theta(\mathrm{deg})$ & $b_{f-c g}(\mathrm{~mm})$ & $b_{w-c g}(\mathrm{~mm})$ & $s_{c g}(\mathrm{~mm})$ \\
\hline $45^{\circ}$ & 68.02 & 1055.74 & 777.04 \\
\hline $55^{\circ}$ & 508.95 & 916.88 & 1036.36 \\
\hline $60^{\circ}$ & 680.12 & 868.65 & 1120.36 \\
\hline $64^{\circ}$ & 840 & 830.32 & 1200 \\
\hline $65^{\circ}$ & 870 & 826.48 & 1217.84 \\
\hline $75^{\circ}$ & 1158.08 & 776.46 & 1364.12 \\
\hline $85^{\circ}$ & 1458.00 & 752.87 & 1499.00 \\
\hline $90^{\circ}$ & 1560 & 750 & 1560 \\
\hline
\end{tabular}

Table 4. Design variable values for transverse plane bulkhead with varied stiffeners distance

\begin{tabular}{ccccccccccc}
\hline $\begin{array}{c}\text { Bulkhead } \\
\text { number }\end{array}$ & $\begin{array}{c}\text { Construction } \\
\text { types }\end{array}$ & \multicolumn{6}{c}{ Distance between stiffeners (mm) } \\
\hline Bulkhead no. 1 & Direct welding & 400 & 500 & 600 & 700 & 800 & 900 & 1000 & 1200 & 1400 \\
\hline Bulkhead no. 2 & Tied by Bracket & 400 & 500 & 600 & 700 & 800 & 900 & 1000 & 1200 & 1400 \\
\hline
\end{tabular}

\subsection{Sloshing pressure in the tank}

According to NK Rules, 2016, Part CSR-T, Section 7/4.2.2.1 [5], the sloshing pressure on the ship longitudinal bulkhead as a result of longitudinal liquid motion can be calculated using Eq. 1 below:

$$
\int P_{s \mathrm{lg}-\ln g}=\rho \cdot g \cdot l_{s l h} \cdot f_{s l h}\left[0.4-\left(0.39-\frac{1.71 l_{s l h}}{L_{C S R-T}}\right) \frac{L_{C S R-T}}{350}\right]
$$

where:

$\rho \quad=$ fluid density in the tank $=0.797 \mathrm{ton} / \mathrm{m}^{3}$

$g \quad=$ gravitational force $=9.81 \mathrm{~m} / \mathrm{s}^{2}$

$h_{\text {fill }}=$ height of cargo, maximum height of the cargo for sloshing is $95 \%$ of maximum height of tank

$=95 \% \times 10.15 \mathrm{~m}=9.642 \mathrm{~m}$

$h_{\max }=$ maximum height of tank $=10.15 \mathrm{~m}$

$f_{\text {slh }}=$ sloshing coefficient (Eq. 2)

$$
=1-2\left(0.7-\frac{h_{\text {fill }}}{h_{\max }}\right)^{2}
$$

$$
=1-2\left(0.7-\frac{9.6425}{10.15}\right)^{2}=0.875
$$

$L_{C S R-T}=\mathrm{LWL}$ on scantling draught position $=152.295$ $\mathrm{m}$

$n_{\text {wash-t }}=$ number of wash bulkhead in tank which is none

$n_{w f} \quad=$ number of web frame in tank which is 7

$a_{\text {wash-t }}=0$, because no wash bulkhead

$\mathrm{a}_{\mathrm{wf}}=$ coefficient of web frame in tank which is 0.572

$f_{w f}=$ factor of sum of web frame and wash bulkhead in tank

$=\mathrm{n}_{\mathrm{wf}}\left(1+\mathrm{n}_{\text {wash }-\mathrm{t}}\right)=7(1+0)=7$

ltk-h $=$ length of tank $=21.75 \mathrm{~m}$

lslh =effective length of sloshing in tank (can be calculated using Eq. 3)

$=\left(\frac{\left(1+n_{\text {wash }-t} \times a_{\text {wash }-t}\right)\left(1+f_{w f} \times a_{w f}\right) l_{t k-h}}{\left(1+n_{\text {wash }-t}\right)\left(1+f_{w f}\right)}\right)$ 


$$
\begin{aligned}
& =\left(\frac{(1+0 \times 0)(1+7 \times 0.572) 21.75}{(1+0)(1+7)}\right) \\
& =13.604 \mathrm{~m}
\end{aligned}
$$

Hence, sloshing pressure in tank is:

$$
\begin{aligned}
P_{\text {slg-lng } g}= & 0.797 \times 9.81 \times 13.604 \times 0.875 \times \\
& {\left[0.4-\left(0.39-\frac{1.71 \times 13.604}{152.195}\right) \frac{152.195}{350}\right] } \\
= & 25.62 \mathrm{kN} / \mathrm{m}^{2}
\end{aligned}
$$

Based on the rules from Nippon Kaiji Kyokai classification bureau, minimum value for sloshing pressure in tank is $20 \mathrm{kN} / \mathrm{m}^{2}$. Sloshing pressure value to be used is the result value from calculation which is $25.62 \mathrm{kN} / \mathrm{m}^{2}$. The sloshing pressure will be evenly distributed on the whole surface of the corrugated bulkhead.

\subsection{Additional load of construction and equipment weight}

Apart from sloshing pressure, the bulkhead structure gets an additional load of construction and equipment weight. The additional weight can be calculated using Eq. 4 from Nippon Kaiji Koyokai (NK) Rules, 2016, Part CSR-T, Section 7/2.2.5.1 [5] as follows:

$$
F_{\text {stat }}=m_{u n} \times g
$$

where:

$$
\begin{array}{ll}
m_{u n} & =\text { weight of unit } \\
g & =\text { gravitational acceleration }
\end{array}
$$

Referring to the Nippon Kaiji Koyokai classification bureau rules, minimum construction weight and equipment working on the bulkhead is 20 ton. Accordingly, the additional weight working on the bulkhead construction is:

$$
\begin{aligned}
\text { Fstat } & =20 \text { ton } \times 9.81 \mathrm{~m} / \mathrm{s}^{2} \\
& =196.2 \mathrm{kN} \text { or } 196200 \mathrm{~N}
\end{aligned}
$$

The above weight is a concentrated load. The average load which is working on the plate surface is varying according to the length of the corrugated plate as well as the corrugation angle.

\section{Result and discussion}

\subsection{The weight of the corrugated bulkhead}

The determination of the optimum corrugated bulkhead form must be based on the weight of the bulkhead structure with varied corrugation angle. The optimum bulkhead weight can be obtained by varying the bulkhead thickness which have the same stress on every corrugation angle. The stress indicator value in this analysis is the von Misses stress of $150 \mathrm{~N} / \mathrm{mm}^{2}$. Von Misses stress is a resultant stress of two different forces which is working on a structure in different direction. In the corrugated bulkhead structure, the two forces are sloshing pressure in z-axis direction and the additional load of construction and equipment weight in yaxis direction.

Once the optimum bulkhead thickness is known, the bulkhead weight can be calculated as well by multiplying the bulkhead volume with steel density which is $7.85 \mathrm{ton} / \mathrm{m}^{3}$ for common steel. Stools weight (upper and lower stool) is then added to the bulkhead weight as an integrated component of the bulkhead.

The result shows that for $45^{\circ}$ of corrugation angle, the bulkhead weight is $\mathbf{5 2 . 8 5}$ ton. If the corrugation angle was increased to $55^{\circ}$, the bulkhead weight is reduced into 41.93 ton (20.67\% of weight reduction). If the corrugation angle was increased to $60^{\circ}$, the bulkhead weight only reduced to 41.18 ton $(1.79 \%$ of weight reduction). This corrugation angle is the maximum angle for the corrugated weight reduction because from $65^{\circ}$ to $90^{\circ}$ of corrugation angles, the corrugated weight is increased with the percentage of $3.79 \%$ $-16.45 \%$.

Hence, minimum weight of corrugated bulkhead can be achieved if the corrugation angle is $60^{\circ}$ while maximum weight on the corrugation angle is $90^{\circ}$. Minimum weight of corrugated bulkhead on $60^{\circ}$ of corrugation angle can be obtained as the plate thickness to hold the same stress on each corrugation angle variation is the smallest. On $60^{\circ}$ of corrugation angle, the stress concentration on the corner of face plate and web plate of the corrugated plate geometry can be minimized. The effect of stress concentration can be clearly seen on the $45^{\circ}, 85^{\circ}$, and $90^{\circ}$ of corrugation angles as it requires more plate thickness to hold the same stress as on the $60^{\circ}$ of corrugation angle. On these angles, the corner of the face plate and web plate is almost broken to hold the stress.

In general, for the same stress value, the changing of corrugation angles has changed the corrugation bulkhead weight significantly (Fig. 4).

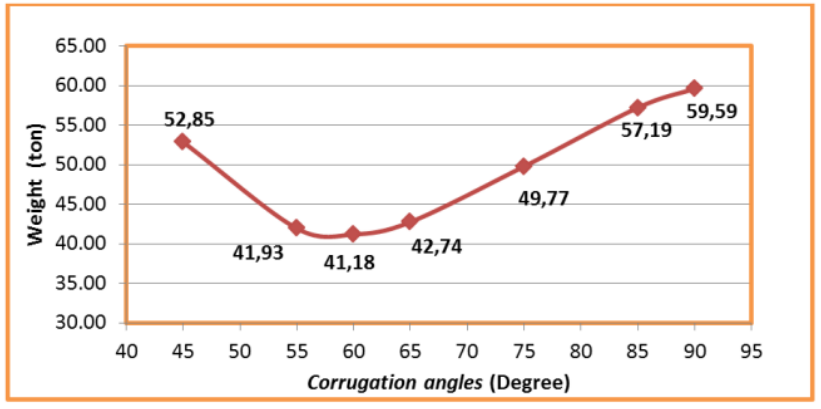

Figure 4. The relationship between corrugation angles and corrugation bulkhead weight

The difference weight between the minimum weight of corrugation bulkhead on $60^{\circ}$ of corrugation angle with other corrugation angles is in the range of $0.75-18.42$ ton. The weight reduction value is only for 1 (one) bulkhead. For 
some bulkheads application in a ship such as tanker ship which requires more bulkheads for its cargo hold, the careful bulkhead selection will give a significant reduction in terms of cost as well the weight reduction.

\subsection{The weight of the transverse plane bulkhead}

For transverse plane bulkhead, two alternatives of bulkhead constructions have been tried out. The first type is transverse plane bulkhead with direct welding while the second type is the transverse plane bulkhead tied by some brackets. The difference between those two alternatives is laying on the integration method of the bulkhead and its stiffeners to the inner bottom plate and deck plate. For direct welding, the bulkhead and its stiffeners are directly welded into inner bottom plate and deck plate. While on the other alternative, stiffeners and brackets is first welded together. The brackets and the bulkhead plate are then welded into inner bottom plate and deck plate.

The stiffeners and brackets dimension are all the same with angle bar or profile L for stiffeners. The bulkhead analysis was done with nine variations of stiffeners distances. Hence, the number of stiffeners on each variation is different.

With the same von Misses stress of $150 \mathrm{~N} / \mathrm{mm}^{2}$ applications as an indicator, the bulkhead thickness has been simulated on each stiffeners distance variation. Based on the simulation, the minimum bulkhead thickness for certain stiffeners distance can be obtained. Hence, the bulkhead weight can be calculated as well by multiplying it with steel density of $7.85 \mathrm{ton} / \mathrm{m}^{3}$. Fig. 5 shows the relationship between stiffeners distance and the bulkhead weight.

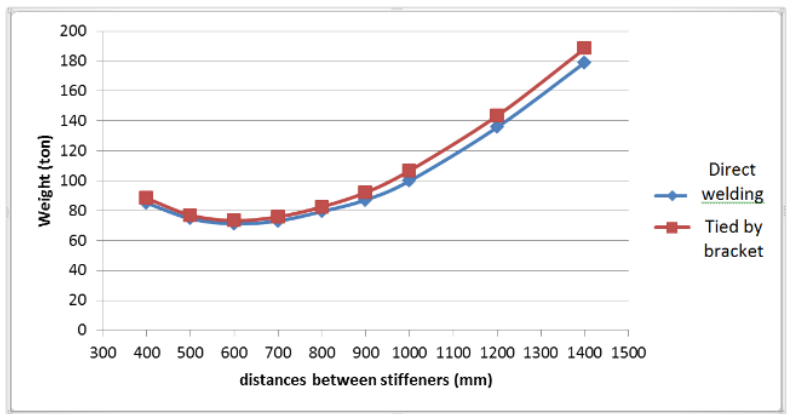

Figure 5. The relationship between stiffeners distance and the bulkhead weight

In Fig. 5, it can be seen that from 400-600 $\mathrm{mm}$ of the distance between stiffeners, the bulkhead weight is decreasing but when the distance between stiffeners is increasing, the bulkhead weight increases as well. The increasing tendency of bulkhead weight from $700 \mathrm{~mm}$ distance between stiffeners was caused by the increasing plate thickness to hold the von Misses stress. From Fig. 5, the optimum distance between stiffeners for minimum weight of the bulkhead is $600 \mathrm{~mm}$.

From the same figure, the total weight value of bulkhead with direct welding is 71.175 ton. This value is smaller $3.03 \%$ if compared with the total weight value of bulkhead tied by brackets of 73.336 ton.

\subsection{The comparison results of corrugated bulkhead with transverse plane bulkhead}

Minimum weight of corrugated bulkhead was obtained when the corrugation angle is $60^{\circ}$ while for transverse plane bulkhead, the minimum weight of the bulkhead was obtained when the distance between stiffeners is $600 \mathrm{~mm}$ with direct welding.

The total minimum weight of the corrugated bulkhead is 41.18 ton while for transverse plane bulkhead with direct welding, the total minimum weight is 71.175 ton. The corrugated bulkhead weight is 29.095 ton or $57.86 \%$ smaller than the transverse plane bulkhead weight. This number is for one bulkhead. In the tanker ship, there are 4 (four) cargo holds. If the corrugated bulkhead is used instead of transverse plane bulkhead in the ship, the total weight reduction of the used material plate is significant.

\section{Conclusion}

Based on the analysis, some conclusions can be drawn as follows:

- With the same strength, the minimum weight of corrugated bulkhead can be obtained on $60^{\circ}$ of corrugation angle while for transverse plane bulkhead, minimum weight can be obtained when the distance between stiffeners is $600 \mathrm{~mm}$.

- Corrugated bulkhead weight is smaller for about $57.86 \%$ of the transverse plane bulkhead weight.

\section{References}

[1] Biro Klasifikasi Indonesia. 2014. Volume II. Rules for the Classification and Construction Seagoing Steel Ship. Jakarta: Indonesia.

[2] Eyres, David J. 2001. Ship Construction, University of Plymouth: India.

[3] Taggart, Robert. 1980. Ship Design and Construction. The Society of Naval Architects and Marine Engineers: New York.

[4] Shama, Mohamed. 2013. Buckling of Ship Structures. Alexandria University: Egypt.

[5] Nippon Kaiji Kyokai (NK) Rules. 2016. Rules for The Survey and Construction of Steel Ship: Part CSR-T Common Structural Rules For Double Hull Oil Tankers: Japan.

[6] Rigo, Ph and J-D. Caprase. 2016. Optimization of Ship Structures. University of Liege: Belgium. 